\title{
A Unifying Passivity Framework for Network Flow Control
}

\author{
John T. Wen and Murat Arcak \\ Department of Electrical, Computer, and Systems Engineering \\ Rensselaer Polytechnic Institute \\ Troy, NY 12180. \\ Emails: wen@cat.rpi.edu, arcak@ecse.rpi.edu
}

\begin{abstract}
Network flow control regulates the traffic between sources and links based on congestion, and plays a critical role in ensuring satisfactory performance. In recent studies, global stability has been shown for several flow control schemes. By using a passivity approach, this paper presents a unifying framework which encompasses these stability results as special cases. In addition, the new approach significantly expands the current classes of stable flow controllers by augmenting the source and link update laws with passive dynamic systems. This generality offers the possibility of optimizing the controllers, for example, to improve robustness and performance with respect to time delay, unmodeled flows, and capacity variation.
\end{abstract}

\section{INTRODUCTION}

Network flow is governed by the interconnection between the information sources and communication links through the routing matrix, $R$, as shown in Fig. 1. Packets from each source (with rate $x_{i}$ ) are routed through the links (with the aggregate link rates $y_{\ell}$ ). Each link has a fixed capacity $c_{\ell}$, and based on its congestion and queue size, a link price, $p_{\ell}$, is computed. The link price information is then sent back to each source to regulate the traffic back into the links. Since the links only feed back the price information to the sources that utilize them, we have the relationship:

$$
y=R x, \quad q=R^{T} p
$$

where $x \in \mathcal{R}^{N}$ is the source rate, $y \in \mathcal{R}^{L}$ is the aggregate rate, $p \in \mathcal{R}^{L}$ is the link price, and $q \in \mathcal{R}^{N}$ is the aggregate price. In this paper, we assume that there is no delay in the loop and the link capacity $c$ is a constant vector.

The flow control problem aims to find decentralized source and link control algorithms ( $x$ as a function of $q$, and $p$ as a function of $y$ ) to achieve the following objectives:

- Utilization: Maximize throughput by keeping $y_{\ell}$ near $c_{\ell}$.

- Fairness: All sources have "equitable" shares of capacity.

- Stability: All signals converge to desired equilibrium values.

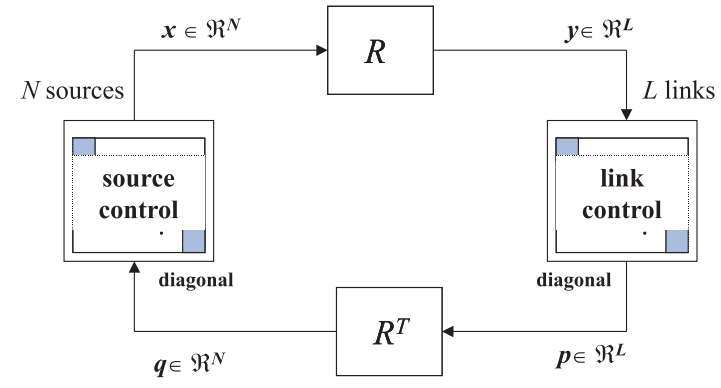

Fig. 1. Network Flow Control Model

- Robustness: Maintain stability and performance under model variation, including time delays, unmodeled flows, and capacity variation.

A common approach to flow control is to decompose the problem into a static optimization problem and a dynamic stabilization problem [1], [2]. The static optimization incorporates fairness, capacity constraint, and utilization, and its solution provides the desired steady state operating point (equilibrium of the closed loop system), $x^{*}, y^{*}, p^{*}$, and $q^{*}$. The source rate and link price update laws are then designed to guarantee stability and robustness of the equilibrium.

The static optimization problem is to maximize the sum of the utilization function $U_{i}\left(x_{i}\right)$ for the sources while complying with capacity constraints in the links; that is

$$
\max _{x \geq 0} \sum_{i=1}^{N} U_{i}\left(x_{i}\right) \quad \text { subject to } \underbrace{R x}_{y} \leq c .
$$

By using the Lagrange multiplier, $p$, the inequality constraint can be folded into the optimization problem:

$$
\begin{aligned}
& \min _{p \geq 0} \max _{x \geq 0} L(x, p) \\
& =\min _{p \geq 0} \max _{x \geq 0} \sum_{i=1}^{N} U_{i}\left(x_{i}\right)-\sum_{\ell=1}^{L} p_{\ell}\left(y_{\ell}-c_{\ell}\right) .
\end{aligned}
$$


If $U$ is differentiable, the first order condition for the maximization problem is

$$
q_{i}=U_{i}^{\prime}\left(x_{i}\right)
$$

The condition for the Lagrange multiplier $p$ is

$$
p_{\ell} \begin{cases}=0 & \text { if } y_{\ell}<c_{\ell} \\ \geq 0 & \text { if } y_{\ell}=c_{\ell} .\end{cases}
$$

If $U$ is strictly concave, (3)-(4) is sufficient to determine a unique equilibrium condition.

The utility function $U_{i}\left(x_{i}\right)$ for each source determines the equilibrium condition, and consequently the steady state fairness and utilization. The objective of source and link update laws is now to drive the actual source rates and link prices to their respective equilibrium values. The constraints in this problem are:

- Decentralization: $x_{i}$ can only depend on $q_{i}$, and $p_{\ell}$ can only depend on $y_{\ell}$.

- No routing information: The routing matrix $R$ is unknown to the sources and the links.

- No coordination among sources and links: The sources do not have knowledge of the objective functions of other sources, and the links do not have information of the capacities of other links. Therefore, the equilibrium value is unknown.

Among many congestion control methods surveyed in [3]-[5], only a few can ensure global stability subject to the structural and information constraints described above. Motivated by the gradient update for the optimization problem (2), a "primal" algorithm is proposed in [1] which consists of a first order source update law and a static link penalty function to keep the aggregate rate below its capacity. A "dual" algorithm is also proposed in [1] where the static source update is the optimality condition (3) and the link uses a first order dynamics of the price update. In [6], [7], the link update is replaced by second order dynamics. Other strategies consider only the first order queue dynamics [8]-[10].

In this paper, we develop a unifying framework for stable network flow control by using the passivity approach [11]. This framework includes the primal and dual control schemes in [1], [7] as special cases and extends them to broader classes of flow control laws. The passivity concept is motivated by physical systems that conserve or dissipate energy, for example, passive circuits [12] and mechanical structures [13]. Passivity provides a useful tool in nonlinear stability analysis and control design for feedback systems [14], [15]. The main result is the celebrated passivity theorem which states that the negative feedback connection of two passive systems is passive. The storage functions (generalization of energy in physical systems) for the subsystems in the feedback interconnection can be combined and used in the Lyapunov stability analysis [16]. By using the passivity approach, we show in this paper that the first order source controller in the primal approach in [1], and the first or second order link controllers in the dual approach in [1], [7], [17] can be replaced by dynamic systems with prescribed passivity properties. In addition, the static link update in [1] and the static source update in [7], [17] can be augmented with a class of dynamic systems motivated by the Zames-Falb multiplier [18]. The dynamic source update in the primal controller [1] can also be combined with the dynamic link update in the dual controller [7] to obtain a dynamic-source/dynamic-link stabilizing control law. The single bottleneck congestion control algorithm in [9] is a special case of this controller.

In addition to unifying the existing stabilizing controllers in the literature, our result also offers the potential for additional optimization for robustness with respect to time delay, unmodeled flows, and capacity variation. Time delay adds phase lag and compromises stability in a feedback system [19]. From the linear system point of view, to enhance robustness with respect to the delay, the controller should add phase lead or increase gain roll-off or do both (subject to the gainphase relationship imposed by the Bode integral formula [19]). The controllers in this paper can provide additional gain roll-off which may be exploited for robustness enhancement. Another consideration is network variation. The model considered in this paper is highly idealized; real networks have constantly changing topology and capacity. For the actual deployment of congestion control, averaging is used to smooth out the variation in various signals. Within our passivity framework, a smoothing filter can be designed and optimized without compromising stability.

This paper is organized as follows. Section II discusses the passivity interpretation of the primal controller in [1] and the generalization to passive source control and multiplier-based link control. Section III presents the passivity framework for the dual controller in [7] and the generalization to passive link control and multiplierbased source control. A combined primal/dual algorithm is also shown in this section. Simulation results based on a four-source/three-link network from are shown in Section IV. Conclusion and future work are given in Section V. Due to the space limitation, the readers are referred to the complete version [20] for most of the proofs.

Notation: Given a $x$ and $f(x)$, the positive projection of $f$ is defined as follows:

$$
(f(x))_{x}^{+} \triangleq \begin{cases}f(x) & \text { if } x>0, \text { or } \\ 0 & \text { if } x=0 \text { and } f(x) \geq 0 \\ 0 & \text { and } f(x)<0 .\end{cases}
$$

If $x$ and $f(x)$ are vectors, then $(f(x))_{x}$ is interpreted 
in the component-wise sense. When $(f(x))_{x}^{+}=0$, we say the projection is active. When $(f(x))_{x}^{+}=f(x)$, the projection is inactive.

\section{Primal Flow Control Algorithms}

In [1], a flow control law based on the primal approach to the optimization problem (2) is proposed (see Fig. 2). Given the utility function for each source, $U_{i}\left(x_{i}\right)$, the source update law is given by

$$
\dot{x}=K\left(U^{\prime}(x)-q\right)_{x}^{+}
$$

where $K=\operatorname{diag}\left\{k_{i}\right\}, k_{i}>0, U^{\prime}(x) \in \mathcal{R}^{N}$ with $i$ th component $U_{i}^{\prime}\left(x_{i}\right)$. The link control is given by

$$
p=h(y)
$$

where $h(y) \in \mathcal{R}^{L}$, with $\ell$ th component $h_{\ell}\left(y_{\ell}\right)$, is a penalty function that enforces the link capacity constraint, $y_{\ell} \leq c_{\ell}$. The equilibrium condition is computed from

$$
q^{*}=U^{\prime}\left(x^{*}\right), \quad p^{*}=h\left(y^{*}\right),
$$

which approximately satisfies the desired condition (3)(4). We assume that each penalty function is monotonically non-decreasing, such as the following used in [1]:

$$
h_{\ell}(y)=\left(y-c_{\ell}+\epsilon\right)^{+} / \epsilon^{2} .
$$

In order for the equilibrium condition to make physical sense, we also assume $x^{*}>0$. It can be shown that the positive projection in (5) does not change the equilibrium of (5)-(6).

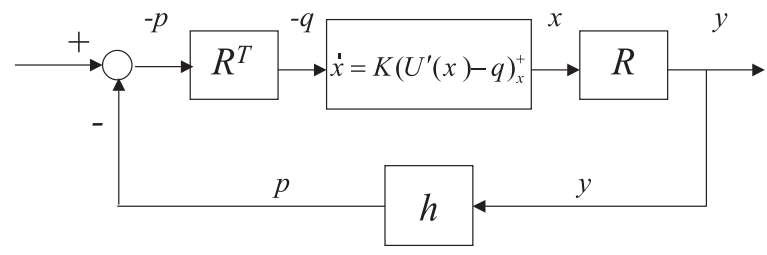

Fig. 2. Primal Flow Controller

\section{A. Passivity Based Stability Analysis}

We now present a stability argument by using the passivity analysis. The advantage of this approach is that it leads to an extended class of stabilizing control laws to be discussed in Section II-B.

We express the interconnected system in Fig. 2 in an equivalent form in Fig. 3 based on the deviation from the equilibrium condition, and rewrite the link update as

$$
h_{1}\left(y-y^{*}\right) \triangleq h\left(y-y^{*}+y^{*}\right)-h\left(y^{*}\right) .
$$

The following result shows that both the forward and return systems are passive [20].

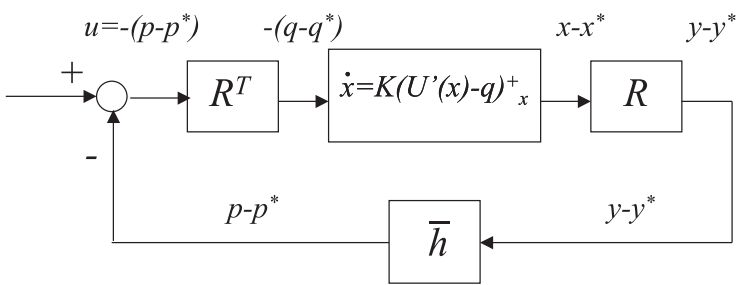

Fig. 3. Equivalent Representation of Primal Flow Controller

Proposition 1: Consider the feedback interconnection shown in Fig. 3. The forward system from $-\left(p-p^{*}\right)$ to $\dot{y}$, and the return system from $\dot{y}$ to $p-p^{*}$ are both passive. Furthermore, the equilibrium $x=x^{*}$ is globally asymptotically stable.

\section{B. An Extended Class of Source Rate Control Laws}

The advantage of the passivity perspective is that it allows us to consider a broader class of stabilizing source control laws. A natural generalization is to replace the first order update (5) by a more general class of passive systems. To motivate this extension, we express (5) as a feedback system as in Fig. 4(a) where

$$
g_{1}\left(x-x^{*}\right) \triangleq-U^{\prime}\left(x-x^{*}+x^{*}\right)+U^{\prime}\left(x^{*}\right) .
$$

Let us now replace the integrator in the forward system by $\frac{1}{s}\left(D+C(s I-A)^{-1} B\right)$ where $(A, B, C, D)$ is a positive real (PR) transfer function (for the definition of positive realness, see [16]), as in Fig. 4(b). This system can be transformed to an equivalent system as shown in Fig. 4(c). The forward system is now passive by design, and the feedback system is also passive since $g_{1}$ is a first/third-quadrant function. It then follows that the system from $-\left(q-q^{*}\right)$ to $\dot{x}$ is passive.

The above argument does not take into account the non-negativity restriction on $x$. The following theorem shows that for certain class of $U(x)$, the source rates would remain positive and the overall system is globally asymptotically stable.

Theorem 1: Consider the source rate control law for source $i, i=1, \ldots, N$, given by

$$
\begin{aligned}
& \dot{\xi}_{i}=A_{i} \xi_{i}+B_{i}\left(U_{i}^{\prime}\left(x_{i}\right)-q_{i}\right), \quad \xi_{i} \in \mathcal{R}^{n_{i}} \\
& \dot{x}_{i}=C_{i} \xi_{i}+D_{i}\left(U_{i}^{\prime}\left(x_{i}\right)-q_{i}\right),
\end{aligned}
$$

where $\left(A_{i}, B_{i}, C_{i}, D_{i}\right)$ is strictly positive real (SPR), the link update given by (6), and the routing connection given by (1).

Assume $U_{i}\left(x_{i}\right) \rightarrow-\infty$ as $x_{i} \rightarrow 0$ for $i=$ $1, \ldots, N$. Then the equilibrium of the interconnected system is globally asymptotically stable, that is, solutions converge to the equilibrium from any initial condition $(x(0), \xi(0)) \in \mathcal{X} \times \mathcal{R}^{n_{1}} \times \ldots \mathcal{R}^{n_{N}}, \mathcal{X} \triangleq$ $\left\{x: x_{i}>0, i=1, \ldots, N\right\}$. 


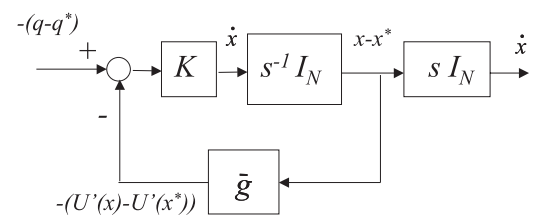

(a)

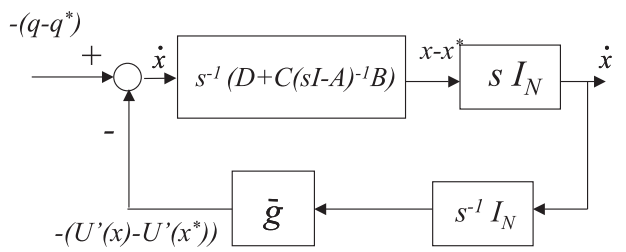

(b)

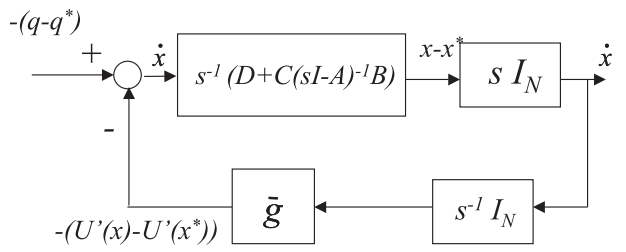

(c)

Fig. 4. Extension of Source Rate Update in Primal Flow Controller

As we show in the proof (see Appendix I), the assumption on $U_{i}$ ensures that $\mathcal{X}$ is positive-invariant, i.e., if $x$ is initially in $\mathcal{X}$, it will remain in $\mathcal{X}$ for all $t \geq 0$. For commonly used utility functions such as $U_{i}\left(x_{i}\right)=a_{i} \log \left(x_{i}\right)$ (proportional fair, also TCP Vegas) and $U_{i}\left(x_{i}\right)=-a_{i} / x_{i}$ (Variant of TCP Reno) [5], this assumption is satisfied. However, for $U_{i}\left(x_{i}\right)=$ $a_{i} \tan ^{-1}\left(b x_{i}\right)$ (TCP Reno), the assumption does not hold. In this case, we impose positive projections on (11)-(12):

$$
\begin{aligned}
& \dot{\xi}_{i}=\left(A_{i} \xi_{i}+B_{i}\left(U_{i}^{\prime}\left(x_{i}\right)-q_{i}\right)\right)_{\xi_{i}}^{+} \\
& \dot{x}_{i}=\left(C_{i} \xi_{i}+D_{i}\left(U_{i}^{\prime}\left(x_{i}\right)-q_{i}\right)\right)_{x_{i}}^{+},
\end{aligned}
$$

and restrict $\left(A_{i}, B_{i}, C_{i}, D_{i}\right)$ to

$$
\begin{aligned}
A_{i} & =\left[\begin{array}{ccc}
-a_{i 1} & & 0 \\
& \ddots & \\
0 & & -a_{i n_{i}}
\end{array}\right] \quad B_{i}=\left[\begin{array}{c}
b_{i 1} \\
\vdots \\
b_{i, n_{i}}
\end{array}\right] \\
C_{i} & =\left[\begin{array}{lll}
c_{i 1} & \ldots & c_{i, n_{i}}
\end{array}\right] \quad D_{i}=d_{i}
\end{aligned}
$$

where $a_{i j}, b_{i j}, c_{i j}$, and $d_{i}$ are positive constants. This restriction eliminates the possibility of phase lead controllers and is therefore of limited utility in terms of enhancing robustness with respect to time delays.

\section{An Alternate Class of Source Rate Control Laws}

The passivity argument in Section II-A uses the fact that the forward system cascaded with the derivative block and the return system pre-multiplied by the integral block are both passive (as shown in Fig. 3). In passivity theory, the derivative block is a special case of multipliers [18] which means that its inverse (integral block) preserves the passivity of the return system. However, this approach limits the possibility of applying a much broader class of multipliers (called Zames-Falb multipliers [21]) to the return system to preserve its passivity property. In this section, we use an alternative passivity argument to show that the forward system is passive without the addition of the derivative block. This will then allow us to use dynamic link controllers and still achieve global asymptotic stability.

The following result shows that $-\left(p-p^{*}\right)$ to $y-y^{*}$ is strictly passive (instead of $-\left(p-p^{*}\right)$ to $\dot{y}$ as in Section IIA).

Proposition 2: Consider the feedback interconnection shown in Fig. 2. The system from $-\left(p-p^{*}\right)$ to $y-y^{*}$ is strictly passive.

As in Section II-B, we can also generalize the first order control law (5) to a broader class of passive systems:

$$
\begin{aligned}
\dot{\xi} & =(A \xi-B x)_{\xi}^{+} \\
\dot{x} & =\left(C \xi+D\left(U^{\prime}(x)-q\right)\right)_{x}^{+}
\end{aligned}
$$

where $(A, B, C, D)$ is as in Theorem 1 . However, since $\xi$ always decays to zero, after the initial transient, this controller is essentially the same as the first order controller (5).

In the context of passivity-based stability analysis, [21] and [18] showed that a monotone first-third quadrant nonlinearity cascaded with a class of transfer functions is passive. To use the result in link control, we first define the following class of transfer functions:

Definition 1: A proper rational function $H(s)$ is called an inverse-ZF (Zames-Falb) function if

$$
H(s)=\left(m_{0}-Z(s)+\eta s\right)^{-1}
$$

where $m_{0}, \eta$ are positive constants and the impulse response of $Z(s), z(t)$, satisfies

$$
z(t)>0, \quad \int_{0}^{\infty} z(t)<m_{0} .
$$

Examples of inverse-ZF functions include first-order lag filters, $1 /\left(a_{0} s+a_{1}\right)$ and $(a s+1) /(s+a), 0 \leq a<1$. We can now extend the static link update Theorem 2 to the penalty function cascaded with an inverse-ZF function. The main idea is to show that when the link penalty function is modified by inverse-ZF functions as in Fig.5(a), it is equivalent to the same modification applied to the error system as in Fig. 5(b).

Theorem 2: If the source update law is given by (5) 


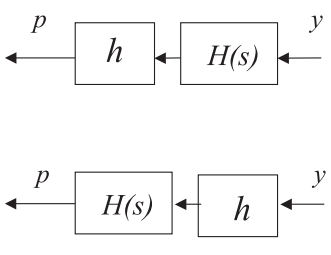

(a)
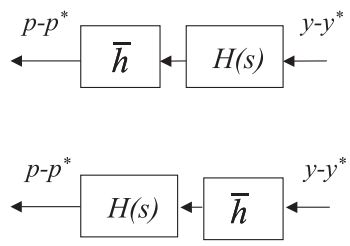

(b)
Fig. 5. Link Controller Modified by Inverse-ZF Functions

and the update law for the lth link is replaced by

$$
\begin{aligned}
& p_{\ell}=h_{\ell}\left(H_{\ell}(s) y_{\ell}\right), \quad \text { or } \\
& p_{\ell}=H_{\ell}(s) h_{\ell}\left(y_{\ell}\right)
\end{aligned}
$$

where $H_{\ell}(s)$ is an inverse-ZF function and $H_{\ell}(0)=1$, then the equilibrium of the closed loop system is the same as in (7) and is globally asymptotically stable.

In (18), $p$ is non-negative since the output of $h$ is nonnegative. However, in (19), this is no longer guaranteed. Since $p$ represents only information feedback rather than physical transmission rate as in $x$, negative values of $p$ may be acceptable.

\section{Dual Flow Control Algorithms}

The flow control algorithm in [1] is based on the primal optimization in (2) and the link rate constraint is enforced by using the penalty function (6). Though the approach guarantees global stability, it does not take the link queue dynamics into account. In [7], a dual approach is proposed where the queue size, $b$, is explicitly modeled and the rate of change of the link price, $\dot{p}$, is determined by the combination of queue size and link rate:

$$
\begin{aligned}
\dot{b} & =(y-c)_{b}^{+} \\
\dot{p} & =\Gamma(\Lambda b+y-c)_{p}^{+}
\end{aligned}
$$

where $\Gamma=\operatorname{diag}\left\{\gamma_{\ell}\right\}, \gamma_{\ell}>0, \Lambda=\operatorname{diag}\left\{\lambda_{\ell}\right\}, \lambda_{\ell}>0$. The source update is directly given by the primal solution (3):

$$
x=U^{\prime-1}(q)
$$

where the $i$ th component of $U^{\prime-1}(q)$ is $U_{i}^{\prime-1}\left(q_{i}\right)$. The overall closed loop system is shown in Fig. 6. By using a Lyapunov function argument, the global asymptotic stability of the system can be shown [7]. We can also apply the same passivity argument as in Section III-A to show that the link controller from $\left(x-x^{*}\right)$ to $\dot{q}$ and the source controller from $-\dot{q}$ to $x-x^{*}$ are both passive (see Fig. 7 with $g_{1}$ given by (10)); and the stability property follows by combining the storage functions of the two systems.

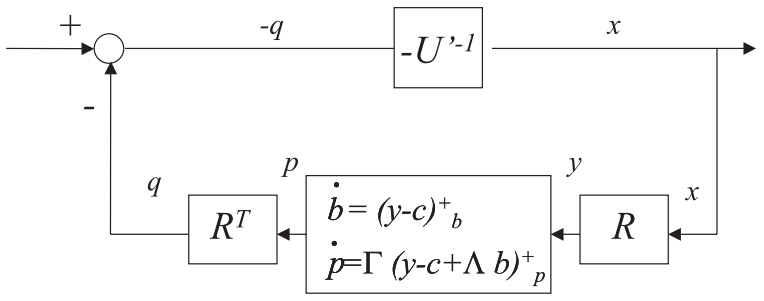

Fig. 6. Dual Flow Controller

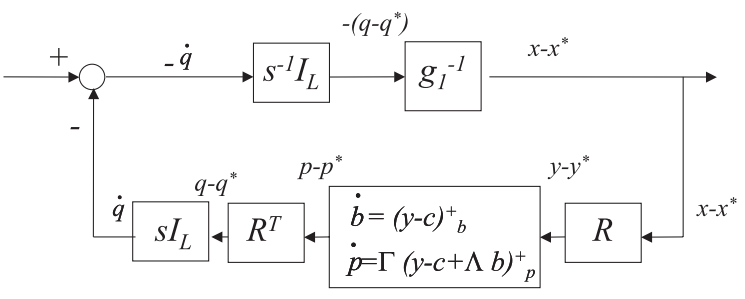

Fig. 7. Equivalent Representation of Dual Flow Controller

\section{A. An Extended Class of Link Price Control Laws}

As in Section II-B, we now extend the second order link update algorithm in (20)-(21) to a larger class of passive dynamic systems. However, note that positive projections are needed in (20)-(21) to ensure the equilibrium condition (4). If the positive projections were removed, the equilibrium condition would imply $y_{\ell}=c_{\ell}$ even for non-bottleneck links, which would in turn lead to unbounded queues for those links. Therefore, as in (15), we restrict the class of SPR filters in the link control law extension. As a motivation of this extension, if we ignore the positive projections, (20)-(21) can be regarded as a first order PR transfer function from $y_{\ell}-c_{\ell}$ to $\dot{p}_{\ell}$ (see Fig. 8(a)). The result below shows that this transfer function can be generalized to a class of PR transfer function (see Fig. 8(b)), and, under suitable positive projection, the closed loop system remains globally asymptotically stable.

Theorem 3: Consider the link update law

$$
\begin{aligned}
& \dot{\eta}=\left(A_{L} \eta+B_{L}(y-c)\right)_{\eta}^{+} \\
& \dot{p}=\left(C_{L} \eta+D_{L}(y-c)\right)_{p}^{+}
\end{aligned}
$$

where $\left(A_{L}, B_{L}, C_{L}, D_{L}\right)$ are block diagonal, partitioned according to each link, with the lth subsystem given by

$$
\begin{aligned}
A_{\ell} & =\left[\begin{array}{ccc}
-a_{\ell 1} & & 0 \\
& \ddots & \\
0 & & -a_{\ell, n_{\ell}}
\end{array}\right] \quad B_{\ell}=\left[\begin{array}{c}
b_{\ell 1} \\
\vdots \\
b_{\ell, n_{\ell}}
\end{array}\right] \\
C_{\ell} & =\left[\begin{array}{lll}
c_{\ell 1} & \ldots & c_{\ell, n_{\ell}}
\end{array}\right] \quad D_{\ell}=d_{\ell}
\end{aligned}
$$

where $a_{\ell m}$ is non-negative, and $b_{\ell m}, c_{\ell m}$ and $d_{\ell}$ are positive constants. Then the equilibrium $(\eta, p)=\left(0, p^{*}\right)$, 


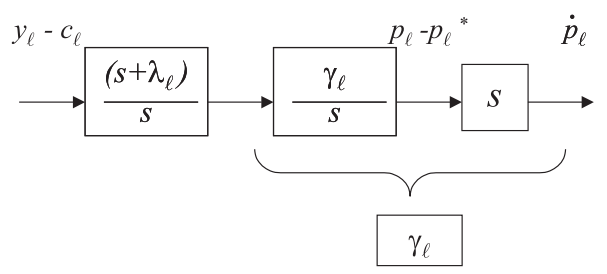

(a)

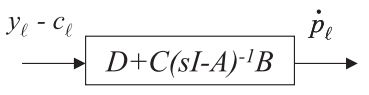

(b)

Fig. 8. Extension of Link Rate Update in Dual Flow Controller

with $p^{*}$ satisfying (4), of the interconnected system described by (22), (23)-(24), and (1) is globally asymptotically stable.

Proof: The proof is given in Appendix II.

Note that the link controller (20)-(21) is a special case of (23)-(24) with $n_{\ell}=1, a_{\ell, 1}=0, b_{\ell, 1}=1, c_{\ell, 1}=\gamma_{\ell} \lambda_{\ell}$, $d_{\ell, 1}=\gamma_{\ell}$. Our extended class also encompasses the first order link control law used in [9], [17]

$$
\dot{p}=\Gamma(y-c)_{p}^{+}
$$

by setting $n_{\ell}=0, c_{\ell, 1}=0, d_{\ell, 1}=\gamma_{\ell}$.

The steady state queue size in (20)-(21) and (26) is zero. Since the queue dynamics is not directly modeled in our extended controller (23)-(24), the steady state queue size may be non-zero. A non-zero queue may be desirable to ensure the full utilization of the link. However, the queue should not be too large in order to avoid excessive queuing delay. If the zero queue size is desired, then at least one of the $a_{\ell, m}$ should be set to zero to emulate the queue dynamics.

\section{B. An Alternate Class of Link Price Control Laws}

In Section II-C, we have shown that the first order primal source control is passive from $-\left(q-q^{*}\right)$ to $x-x^{*}$, and this property allows dynamics to be introduced into the link control by using inverse Zames-Falb functions. In this section, we present the dual result where the link controller is passive from $\left(x-x^{*}\right)$ to $q-q^{*}$.

Proposition 3: Consider the link control law (26). The return system from $y-y^{*}$ to $p-p^{*}$ is passive.

This passivity property can be extended [20] to the adaptive virtue queue link controller in [22]:

$$
\dot{\eta}_{\ell}=\gamma_{\ell}\left(y_{\ell}-c_{\ell}\right)_{\eta_{\ell}}^{+}, \quad p_{\ell}=f_{\ell}\left(y_{\ell}, \eta_{\ell}\right)
$$

where $f$ is non-negative and strictly increasing in both variables. In [10], a nonlinear $\gamma_{\ell}$ is needed to show stability; here we only require $\gamma_{\ell}$ to be a positive constant. The above passivity property does not hold for the second order controller in [7]. This is not surprising since, as shown in Fig. 8(a), if we ignore the positive projection, the transfer function from $y_{\ell}-c_{\ell}$ to $p_{\ell}-p_{\ell}^{*}$ has two poles in the origin, which cannot be PR.

As in Theorem 2, we can make the same modification for the source control by using the inverse-ZF function:

Theorem 4: If the link update law is (26) and the source update law is

$$
\begin{aligned}
& x_{i}=H_{i}(s) U^{\prime}{ }_{i}^{-1}\left(q_{i}\right) \\
& x_{i}=U^{\prime}{ }_{i}{ }^{-1}\left(H_{i}(s) q_{i}\right)
\end{aligned}
$$

where $H_{i}(s)$ is an inverse-ZF function and $H_{i}(0)=1$, then the equilibrium of the closed loop system is the same as in (3)-(4) and is globally asymptotically stable.

Note that in (28), the source rate may become negative making it physically unrealizable.

\section{A Combined Primal/Dual Algorithm}

In Section II-C, we showed passivity of the first order source rate controllers from $-\left(p-p^{*}\right)$ to $\left(y-y^{*}\right)$, and in Section III-B, we showed passivity of the first order link price controller from $\left(y-y^{*}\right)$ to $\left(p-p^{*}\right)$. As a direct consequence of the passivity analysis, we can combine these two classes of controllers together to achieve global asymptotic stability as in Fig. 9. Among the congestion control laws in the literature which guarantee global asymptotic stability, either the source rate update or the link price update is static. When both are dynamic, stability has only been shown using singular perturbation in [10] and for the single-bottleneck case in [8], [9]. With our passivity approach, extension from single to multiple bottlenecks is straightforward because pre-multiplication by $R^{T}$ and post-multiplication by $R$ as in Fig. 9 does not change the passivity property of the forward system.

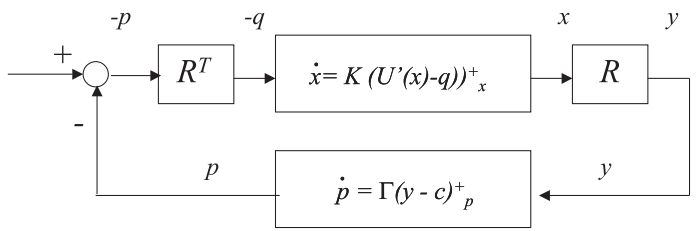

Fig. 9. Primal/Dual Congestion Control

\section{Simulation Results}

To illustrate the new classes of stable flow controllers presented in this paper, we consider a simple 4-source/3link example shown in Fig. 10. The corresponding routing matrix is

$$
R=\left[\begin{array}{llll}
1 & 0 & 1 & 0 \\
1 & 1 & 1 & 0 \\
1 & 1 & 0 & 1
\end{array}\right]
$$


We assume that all source utility functions are $U_{i}\left(x_{i}\right)=$ $\log \left(x_{i}\right)$ and the link capacities are all 1 . For this choice of $U_{i}$, the assumption in Theorem 1 is satisfied. The solution to the optimality condition (3)-(4) is

$$
\begin{aligned}
& x^{*}=\left[\begin{array}{llll}
0.25 & 0.25 & 0.5 & 0.5
\end{array}\right]^{T} \\
& q^{*}=\left[\begin{array}{llll}
4 & 4 & 2 & 2
\end{array}\right]^{T} \\
& y^{*}=\left[\begin{array}{lll}
0.75 & 1 & 1
\end{array}\right]^{T} \\
& p^{*}=\left[\begin{array}{lll}
0 & 2 & 2
\end{array}\right]^{T} \text {. }
\end{aligned}
$$

The initial source rate is set to

$$
x(0)=\left[\begin{array}{llll}
0.4 & 0.6 & 0.8 & 1.0
\end{array}\right]^{T} .
$$

The initial link price is set to zero.

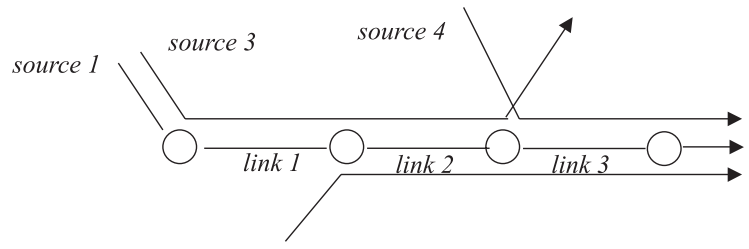

source 2

Fig. 10. A Network Example

To facilitate discussion, we summarize the various flow control laws discussed in this paper in Tables IIII.

\begin{tabular}{|c|c|}
\hline & Combined Primal/Dual Control (Section III-C) \\
\hline source & $\dot{x}=K\left(U^{\prime}(x)-q\right)_{x}^{+}$ \\
\hline link & $\dot{p}=\Gamma(y-c)_{p}^{+}$ \\
\hline
\end{tabular}

TABLE III

Primal/Dual Flow Control LaW

\section{A. Robustness with respect to Time Delays}

1) Dynamic-Source/Static-Link Case: We first show that the robustness with respect to time delays can be enhanced by augmenting the first order source controller in [1] with an SPR filter as in (11)-(12). We use the following controller parameters:

(A1) Flow control in [1] with $k_{i}=0.01$ and $\epsilon=0.1$.

(B1) Flow control in Theorem 1 with $A_{i}=-4, B_{i}=$ 0.04, $C_{i}=-1, D_{i}=0.01$ and $\epsilon=0.1$.

Both controllers work well when no delay is present. However, when a delay of $2 \mathrm{sec}$ is introduced in each link feedback channel, the source rates for controller A2 show persistent oscillations, while for controller B2, they remain well behaved (the source rate comparison is shown in Fig. 11). The loop gain of the linearized system (about the source rate $\bar{x}$ ) is

$$
G_{S}(s)=-s^{-1} W(s)\left(U^{\prime \prime}(\bar{x})-R^{T} h^{\prime}(R \bar{x}) R\right) .
$$

where $W(s)=k_{i}$ for controller $\mathrm{A} 1$, and $W(s)=$ $D_{i}+C_{i}\left(s I-A_{i}\right)^{-1} B_{i}$ for controller B1. The Bode plots of $s^{-1} W(s)$ for both controllers are shown in Fig. 12. It is evident that the SPR filter in B1 adds a significant amount of phase lead, thus improving the stability robustness with respect to the time delay.

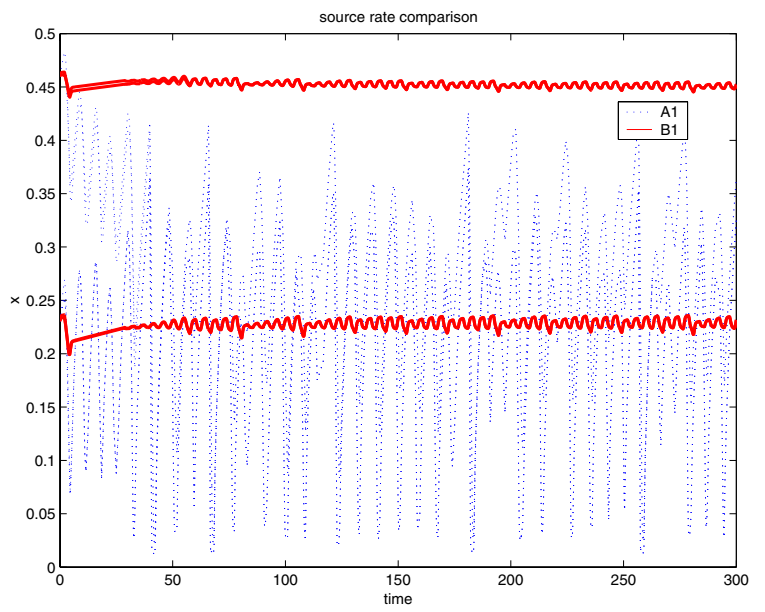

Fig. 11. Source Rate Comparison under 2 sec Delay: Flow Controller in [1] vs. Controller based on Theorem 1
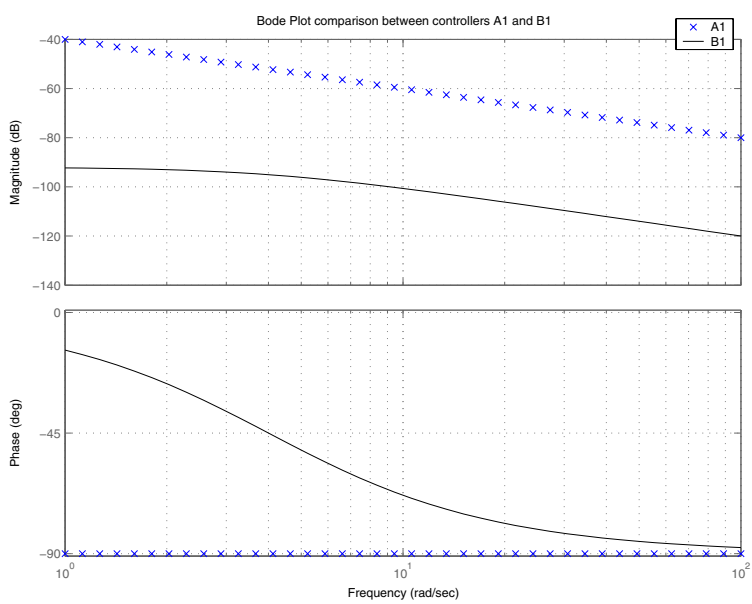

Fig. 12. Loop Gain Bode Plot Comparison: Flow Controller in [1] vs. Controller based on Theorem 1

2) Static-Source/Dynamic-Link Case: We next compare the delay robustness of the second-order link controller in [7] with a modified controller based on Theorem 3:

(A2) Flow control in [7], with $\gamma_{\ell}=2$ and $\lambda_{\ell}=2$. 


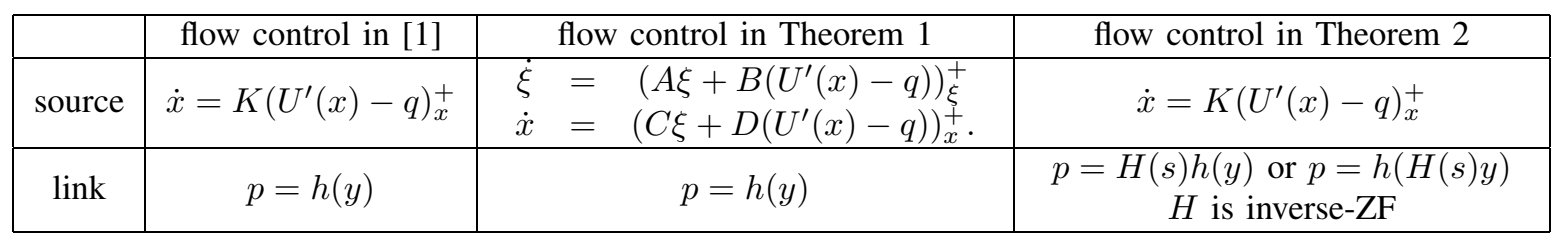

TABLE I

Primal Flow CONTROL LAWS

\begin{tabular}{|c|c|c|c|}
\hline & flow control in [7] & flow control in Theorem 3 & flow control in Theorem 4 \\
\hline source & $x=U^{\prime-1}(q)$ & $x=U^{\prime-1}(q)$ & $\begin{array}{c}x=H(s) U^{\prime-1}(q) \text { or } x=U^{\prime-1}(H(s) q) \\
H \text { is inverse-ZF }\end{array}$ \\
\hline \multirow{2}{*}{ link } & $\begin{array}{c}\dot{b}= \\
\end{array} \quad \begin{array}{c}(y-c)_{b}^{+} \\
\dot{p}=\end{array}(y-c+\Lambda b)_{p}^{+}$. & $\begin{array}{l}\dot{\eta}=(A \eta+B(y-c))_{\eta}^{+} \\
\dot{p}=(C \eta+D(y-c))_{p}^{+} .\end{array}$ & $\dot{p}=\Gamma(y-c)_{p}^{+}$ \\
\hline
\end{tabular}

TABLE II

DuAL Flow CONTROL LAWS

(B2) Flow control in Theorem 3, with $A_{\ell}=-1, B_{\ell}=1$, $C_{\ell}=2, D_{\ell}=2$.

Note that the only difference between (A2) and (B2) is that $A_{\ell}$ is zero in (A2) and negative in (B2). With a constant delay of 1 second introduced in all source to link channels, a persistent oscillation appears with (A2) while a stable response is maintained with (B2), as shown in the link rate comparison in Figure 13. This difference in robustness can again be explained by the phase margin comparison of the linearized system. The loop gain of the linearized system (about the source rate $\bar{x})$ is

$$
G_{L}(s)=-R U^{\prime \prime}(\bar{x})^{-1} R^{T} s^{-1}\left(D_{L}+C_{L}\left(s I-A_{L}\right)^{-1} B_{L}\right) .
$$

Figure 14 shows the Bode plot comparison of the $(1,1)$ element of the loop gain transfer matrix with $\bar{x}=x^{*}$ (other channels are similar). The phase margin corresponding to Controller B2 is much larger than the phase margin of Controller A2, and both controllers have comparable bandwidth. This indicates that B2 should have better performance in the presence of time delays.

\section{B. Disturbances Rejection}

To illustrate the effect of our augmented controller on disturbance rejection, we add a sinusoidal disturbance to the link rate:

$$
y=R x+n_{y}, \quad n_{y}=A \sin (\omega t)
$$

with $A=0.4$ and $\omega=0.4 \pi$. We consider the first order rate controller from [1] and the inverse-ZF augmented controller (last column in Table I) below:

(A3) Flow control in [1] with $k_{i}=0.001$ and $\epsilon=0.1$.

(B3) Flow control in Theorem 2 with $k_{i}=0.001, \epsilon=$ 0.1 , and $H_{\ell}(s)=1 /(s / 0.02+1)$.

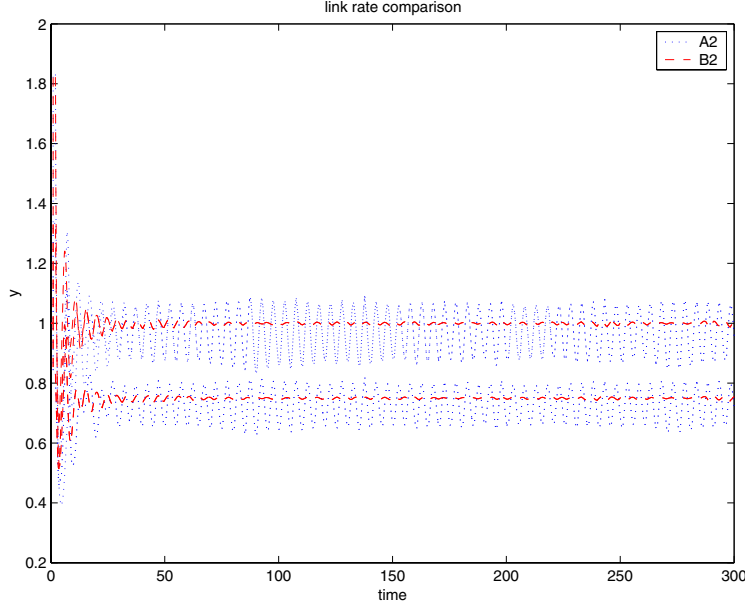

Fig. 13. Link Rate Comparison under $1 \mathrm{sec}$ Delay: Flow Controller in [7] vs. Controller based on Theorem 3

The transfer function of the linearized system (about $\bar{x}$ ) from $n_{y}$ to $x$ and $y$ are

$$
\begin{aligned}
& G_{x, n_{y}}(s)=-G_{H}(s)\left(I+R G_{H}(s)\right)^{-1} \\
& G_{y, n_{y}}(s)=\left(I+R G_{H}(s)\right)^{-1}
\end{aligned}
$$

where

$$
G_{H}(s) \triangleq\left(s I-G_{s}(s) U^{\prime \prime}(\bar{x})\right)^{-1} G_{s}(s) R^{T} h^{\prime}(R \bar{x}) H(s) .
$$

The singular value plots of $G_{x, n_{y}}(s)$ for controllers A3 and B3 in Figure 15 (with $\bar{x}=x^{*}$ ) shows that for disturbance frequency higher than $0.2 \mathrm{rad} / \mathrm{sec}$, we expect B3 to perform better than A3. This is indeed confirmed by the source rate plots shown in Figure 16, in which, after a larger initial transient, B3 removes most of the higher frequency disturbance. Controller B3 also reduces the bandwidth of the closed loop system which has the negative side effect of large transient fluctuations in the 

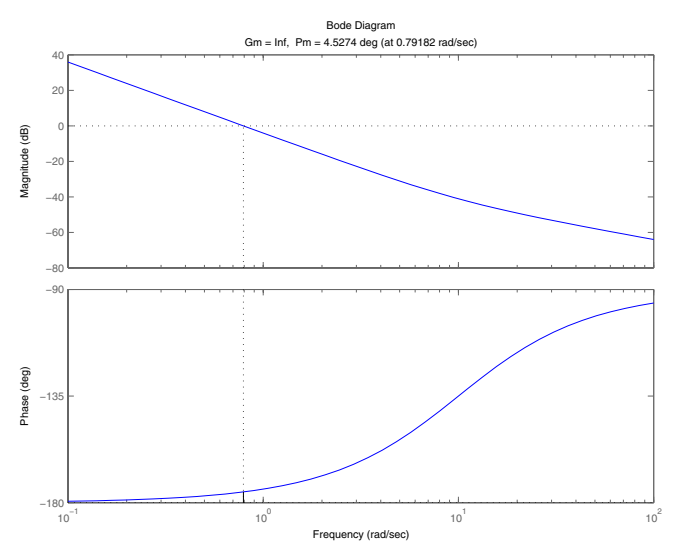

Controller A2

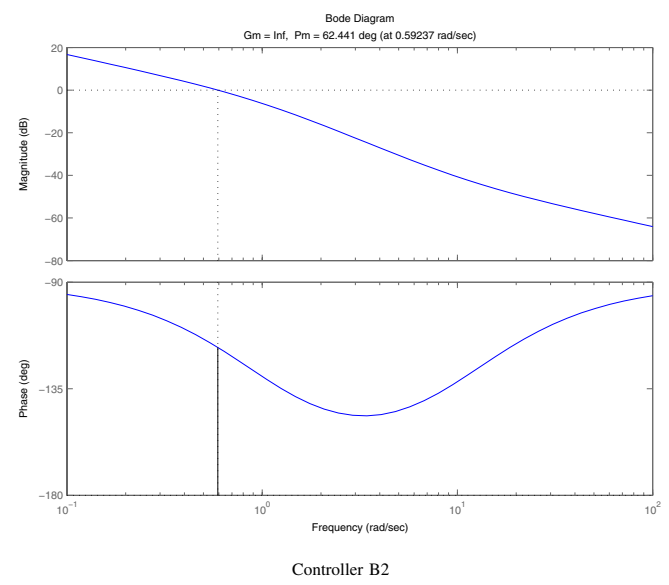

Fig. 14. Bode Plot Comparison of Linearized System: Flow Controller in [7] vs. Controller based on Theorem 3

source rate. Note that the effect of noise on $x$ and $y$ is complementary, i.e., if $n_{y}$ has a small effect on $x$, then it will strongly influence $y$, and vice versa.

\section{CONCLUSION}

Network flow control is commonly decomposed into two steps: a static optimization that incorporates utilization and fairness to establish the desired equilibrium, and dynamic source and link control to ensure stability and robustness of the equilibrium. This paper addresses source and link control which can guarantee global asymptotic stability of the desired equilibrium. By using the passivity approach in nonlinear analysis, we have developed a unifying framework for stabilizing source and link control laws which encompass existing algorithms in [1], [7], [9], [10], [17] as special cases. In our approach, we first interpret the existing results in a passivity perspective and then augment the source and link control laws with suitable passive systems. The global asymptotic stability of the closed loop system then follows from the passivity-based stability analysis. In addition to unifying and extending the existing results, we have shown that the added design freedom in flow

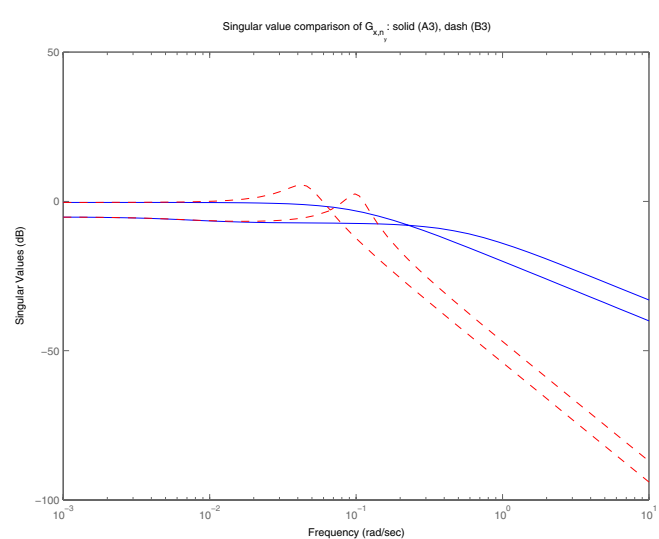

Fig. 15. Singular Value Comparison of $G_{x, n_{y}}(j \omega)$ for Controllers $\mathrm{A} 3$ and $\mathrm{B} 3$

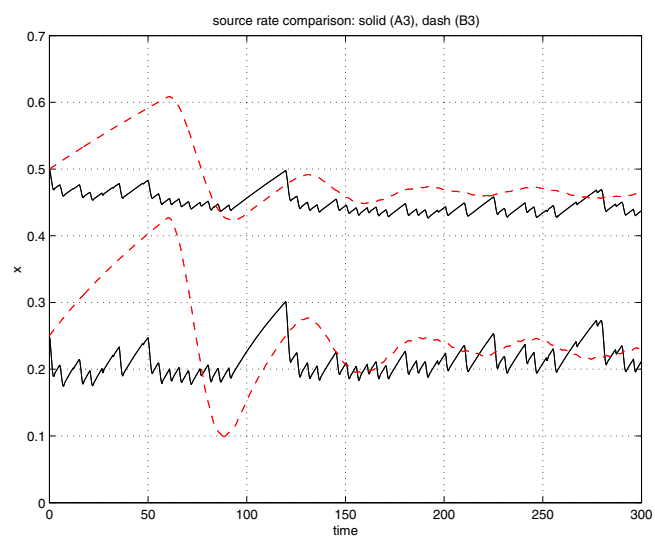

Fig. 16. Source Rate Comparison under Sinusoidal Disturbance for Controllers A3 and B3

control can be exploited to improve the disturbance rejection property and robustness with respect to time delays. The performance improvement and robustness enhancement potential is illustrated through linearized analysis and simulation. We are currently developing a systematic flow control design strategy with this passivity framework and investigating stability and robustness under discrete time implementations.

\section{ACKNOWLEDGMENT}

The authors would like to thank helpful discussion of this research with Shivkumar Kalyanaraman, Ariel Loncarich, and Xingzhe Fan. This research is supported in part by the RPI Office of Research through an Exploratory Seed Grant.

\section{APPENDIX I \\ PROOF OF THEOREM 1}

From [23], [24], $\left(A_{i}, B_{i}, C_{i}, D_{i}\right)$ is SPR if and only if there exists $P_{i}>0, L_{i}>0, Q_{i}, W_{i}$ such that 


$$
\begin{aligned}
P_{i} A_{i}+A_{i}^{T} P_{i} & =-L_{i}-Q_{i}^{T} Q_{i} \\
B_{i}^{T} P_{i}-C_{i} & =W_{i}^{T} Q_{i} \\
W_{i}^{T} W_{i} & =D_{i}+D_{i}^{T} .
\end{aligned}
$$

For the forward system, let

$$
V_{1}\left(x-x^{*}, \xi\right)=\sum_{i}\left(\frac{1}{2} \xi_{i}^{T} P_{i} \xi_{i}-\left(U_{i}\left(x_{i}\right)-U_{i}\left(x_{i}^{*}\right)\right)+q_{i}^{*}\left(x_{i}-x_{i}^{*}\right)\right) .
$$

Since $U_{i}$ is strictly concave, $V_{1}$ is a positive definite function. The derivative of $V_{1}$ along the solution is

$$
\begin{aligned}
\dot{V}_{1}= & \sum_{i}\left[\frac{1}{2} \xi_{i}^{T}\left(P_{i} A_{i}+A_{i}^{T} P_{i}\right) \xi_{i}\right. \\
& \left.+\xi_{i}^{T} P_{i} B_{i}\left(U_{i}^{\prime}\left(x_{i}\right)-q_{i}\right)+\left(-U_{i}^{\prime}\left(x_{i}\right)+q_{i}^{*}\right)^{T} \dot{x}_{i}\right] .
\end{aligned}
$$

Adding and subtracting $q_{i}$ to $q_{i}^{*}$, and using (12) and (32)(34), we get (note that $D_{i}$ and $W_{i}$ are scalars)

$$
\begin{aligned}
& \dot{V}_{1}=\sum_{i} {\left[\frac{1}{2} \xi_{i}^{T}\left(P_{i} A_{i}+A_{i}^{T} P_{i}\right) \xi_{i}\right.} \\
&+\left(C_{i} \xi_{i}+Q_{i} \xi_{i} W_{i}\right)\left(U_{i}^{\prime}\left(x_{i}\right)-q_{i}\right) \\
& \quad\left(U_{i}^{\prime}\left(x_{i}\right)-q_{i}\right)\left(C_{i} \xi_{i}+D_{i}\left(U_{i}^{\prime}\left(x_{i}\right)-q_{i}\right)\right) \\
&\left.\quad-\left(q_{i}-q_{i}^{*}\right) \dot{x}_{i}\right] \\
&=\sum_{i} {\left[-\frac{1}{2} \xi_{i}^{T} L \xi_{i}-\frac{1}{2}\left(Q_{i} \xi_{i}\right)^{2}\right.} \\
&+Q_{i} \xi_{i} W_{i}\left(U_{i}^{\prime}\left(x_{i}\right)-q_{i}\right) \\
&\left.\quad-\frac{1}{2}\left(W_{i}\left(U_{i}^{\prime}\left(x_{i}\right)-q_{i}\right)\right)^{2}-\left(q_{i}-q_{i}^{*}\right) \dot{x}_{i}\right] \\
&=\sum_{i}\left[-\frac{1}{2} \xi_{i}^{T} L \xi_{i}-\frac{1}{2}\left(Q_{i} \xi_{i}-W_{i}\left(U_{i}^{\prime}\left(x_{i}\right)-q_{i}\right)\right)^{2}\right. \\
&\left.\quad-\left(q_{i}-q_{i}^{*}\right) \dot{x}_{i}\right] \\
& \leq \sum_{i}\left[-\sigma_{\min }\left(L_{i}\right)\left\|\xi_{i}\right\|^{2}-\left(q_{i}-q_{i}^{*}\right) \dot{x}_{i}\right] .
\end{aligned}
$$

Because the first term is negative semidefinite, the forward system from $-\left(q-q^{*}\right)$ to $\dot{x}$ is passive.

Now consider the return system, and let

$$
V_{2}\left(y-y^{*}\right)=\sum_{\ell}\left(\int_{y_{\ell}^{*}}^{y_{\ell}}\left(h(\sigma)-h\left(y_{\ell}^{*}\right)\right) d \sigma\right),
$$

Since $h\left(y_{\ell}\right)-h\left(y_{\ell}^{*}\right)$ is first-third quadrant, $V_{2}$ is a nonnegative definite function. The return system from $\dot{x}$ to $q-q^{*}$ is passive since

$$
\dot{V}_{2}=\left(h(y)-h\left(y^{*}\right)\right)^{T} \dot{y}=\left(p-p^{*}\right)^{T} \dot{y}=\left(q-q^{*}\right)^{T} \dot{x} .
$$

We can now use $V=V_{1}+V_{2}$ as a Lyapunov function and obtain

$$
\dot{V} \leq-\sum_{i} \sigma_{\min }\left(L_{i}\right)\left\|\xi_{i}\right\|^{2} .
$$

Since $U_{i}\left(x_{i}\right) \rightarrow-\infty$ as $x_{i} \rightarrow 0$, the level sets of $V$ are confined in $\mathcal{X} \times \mathcal{R}^{n_{1}} \times \ldots \mathcal{R}^{n_{N}}$,
$\mathcal{X} \triangleq\left\{x: x_{i}>0, i=1, \ldots, N\right\}$. Thus, $\mathcal{X}$ is positiveinvariant.

It follows from (35) and LaSalle's Invariance principle (see [16] for a detailed discussion) that $(x(t), \xi(t))$ converges to the largest invariant set contained in

$$
\Omega=\{(x, \xi): \xi=0\} .
$$

On $\Omega, \dot{\xi}_{i}=0$ for all $i$, therefore, from (11), $U_{i}^{\prime}\left(x_{i}\right)=q_{i}$. Because $x=x^{*}$ is the unique equilibrium of (5)-(6), we conclude that $\Omega=\left\{\left(x^{*}, 0\right)\right\}$; that is, $\left(x^{*}, 0\right)$ is globally asymptotically stable.

\section{APPENDIX II}

\section{PROOF OF THEOREM 3}

We first show that the modified return system (23)(24) is passive. Consider the following positive definite function for the $\ell$ th link:

$$
V_{2_{\ell}}=\left\{\sum_{m} \frac{c_{\ell m}}{2 b_{\ell m}} \eta_{\ell m}^{2}\right\}+\left(c_{\ell}-y_{\ell}^{*}\right) p_{\ell} .
$$

The derivative along the solution is (after adding and subtracting $y_{\ell}$ from $y_{\ell}^{*}$ ):

$$
\begin{aligned}
\dot{V}_{2_{\ell}}= & \sum_{m} \frac{c_{\ell m}}{b_{\ell m}} \eta_{\ell m}\left(-a_{\ell m} \eta_{\ell m}+b_{\ell m}\left(y_{\ell}-c_{\ell}\right)\right)_{\eta_{\ell m}}^{+} \\
& +\left(c_{\ell}-y_{\ell}\right)\left(\sum_{m} c_{\ell m} \eta_{\ell m}+d_{\ell}\left(y_{\ell}-c_{\ell}\right)\right)_{p_{\ell}}^{+} \\
& +\left(y_{\ell}-y_{\ell}^{*}\right) \dot{p}_{\ell} .
\end{aligned}
$$

First observe that

$$
\begin{aligned}
& \frac{c_{\ell m}}{b_{\ell m}} \eta_{\ell m}\left(-a_{\ell m} \eta_{\ell m}+b_{\ell m}\left(y_{\ell}-c_{\ell}\right)\right)_{\eta_{\ell m}}^{+} \\
& =-\frac{a_{\ell m} c_{\ell m}}{b_{\ell m}} \eta_{\ell m}^{2}+c_{\ell m} \eta_{\ell m}\left(y_{\ell}-c_{\ell}\right) .
\end{aligned}
$$

This follows because, if the projection is inactive then both sides of the equality are equal, and if the projection is active, $\eta_{\ell m}=0$, and both sides of the equality are zero.

Next we claim that

$$
\begin{aligned}
& \left(c_{\ell}-y_{\ell}\right)\left(\sum_{m} c_{\ell m} \eta_{\ell m}+d_{\ell}\left(y_{\ell}-c_{\ell}\right)\right)_{p_{\ell}}^{+} \\
& \leq\left(c_{\ell}-y_{\ell}\right) \sum_{m} c_{\ell m} \eta_{\ell m} .
\end{aligned}
$$

If the projection is inactive, the inequality holds since $d_{\ell}\left(y_{\ell}-c_{\ell}\right)^{2} \geq 0$. If the projection is active, then

$$
\sum_{m} c_{\ell m} \eta_{\ell m}+d_{\ell}\left(y_{\ell}-c_{\ell}\right) \leq 0
$$

which implies that $\eta_{\ell m}=0$ for all $m$ if $d_{\ell}=0$ or $y_{\ell}-c_{\ell}=0$ if $d_{\ell}>0$; in either case the left hand side of (38) is zero and the right hand side is non-negative. Hence, (38) is true. 
Substitute (37) and (38) in (36), we obtain

$$
\dot{V}_{2 \ell} \leq\left\{\sum_{m}-\frac{a_{\ell m} c_{\ell m}}{b_{\ell m}} \eta_{\ell m}^{2}\right\}+\left(y_{\ell}-y_{\ell}^{*}\right) \dot{p}_{\ell} .
$$

Summing $V_{2 \ell}$ over all links and using the condition (1), it follows that the return system from $-\left(x-x^{*}\right)$ to $\dot{q}$ is passive.

Now consider the forward system, and let

$$
V_{1}\left(q-q^{*}\right)=\sum_{i} \int_{q_{i}^{*}}^{q_{i}}\left(x_{i}^{*}-U_{i}^{\prime-1}(\sigma)\right) d \sigma .
$$

where $V_{1}(0)=0, \nabla V_{1}(0)=\sum_{i}\left(x_{i}^{*}-U_{i}^{\prime-1}\left(q_{i}^{*}\right)\right)=0$, and $\nabla^{2} V_{1}=-\sum_{i} U_{i}^{\prime \prime}{ }^{-1}\left(q_{i}\right)>0$, so $V_{1}$ is a positive definite function. The forward system from $-\dot{q}$ to $x-x^{*}$ is passive since

$$
\dot{V}_{1}=\sum_{i}\left(x_{i}^{*}-U_{i}^{\prime-1}\left(q_{i}\right)\right) \dot{q}_{i}=\sum_{i}\left(x_{i}^{*}-x_{i}\right) \dot{q}_{i} .
$$

We now use $V=V_{1}+V_{2}$ as a Lyapunov function to obtain

$$
\begin{aligned}
\dot{V} \leq \sum_{\ell} & \left\{\sum_{m} c_{\ell m} \eta_{\ell m}\left(y_{\ell}-c_{\ell}\right)\right. \\
& \left.+\left(c_{\ell}-y_{\ell}\right)\left(\sum_{m} c_{\ell m} \eta_{\ell m}+d_{\ell}\left(y_{\ell}-c_{\ell}\right)\right)_{p_{\ell}}^{+}\right\}
\end{aligned}
$$

which is non-positive as shown before. Using the LaSalle Invariance Principle, it follows that $\left(\eta_{\ell m}, p_{\ell}\right)$ converges to the largest invariant set $\Omega$ within the set

$$
\begin{aligned}
& \left\{\left(\eta_{\ell m}, p_{\ell}\right): W\left(\eta_{\ell m}, p_{\ell}\right)=\sum_{m} c_{\ell m} \eta_{\ell m}\left(y_{\ell}-c_{\ell}\right)\right. \\
& \left.+\left(c_{\ell}-y_{\ell}\right)\left(\sum_{m} c_{\ell m} \eta_{\ell m}+d_{\ell}\left(y_{\ell}-c_{\ell}\right)\right)_{p_{\ell}}^{+}=0\right\} .
\end{aligned}
$$

First consider the projection being inactive, $p_{\ell}>0$, then $W\left(\eta_{\ell m}, p_{\ell}\right)=-d_{\ell}\left(y_{\ell}-c_{\ell}\right)^{2}$, and, therefore, $y_{\ell}=$ $c_{\ell}$ on $\Omega$. From (23), it follows that $\eta_{\ell m} \rightarrow 0$ for all $m$ on $\Omega$.

Next consider the projection being active $\left(p_{\ell}=0\right.$ in this case), then on $\Omega, \eta_{\ell m}\left(y_{\ell}-c_{\ell}\right)=0$. The case $y_{\ell}=c_{\ell}$ is the same as before, so consider $\eta_{\ell m}=0$ only. Since $\Omega$ is invariant, $\dot{\eta}_{\ell m}=0$ also. This implies $y_{\ell}-c_{\ell} \leq 0$ or $y_{\ell} \leq c_{\ell}$.

Combining the two cases, we conclude that $\Omega$ consists of the equilibrium $(\eta, p)=\left(0, p^{*}\right)$ where $p^{*}$ satisfies (4).

\section{REFERENCES}

[1] F. Kelly, A. Maulloo, and D. Tan, "Rate control in communication networks: shadow prices, proportional fairness and stability," Journal of the Operational Research Society, vol. 49, pp. 237252, 1998 .
[2] S. Low and D. Lapsley, "Optimization flow control - I: basic algorithm and convergence," IEEE/ACM Transaction on Networking, vol. 7, no. 6, pp. 861-874, 1999.

[3] R. Jain, "Congestion control and traffic management in ATM networks: Recent advances and a survey," Computer Networks and ISDN Systems, vol. 28, no. 13, pp. 1723-1738, Feb. 1995.

[4] R. Srikant, "Control of communication networks," in Perspectives in Control Engineering: Technologies, Applications, New Directions, T. Samad, Ed. IEEE Press, 2000, pp. 462-488.

[5] S. Low, F. Paganini, and J. Doyle, "Internet congestion control," IEEE Control Systems Magazine, vol. 22, no. 1, pp. 28-43, 2002.

[6] S. Athuraliya, S. Low, V. Li, and Q. Yin, "REM: active queue management," IEEE Network, vol. 15, no. 3, pp. 48-53, 2001.

[7] F. Paganini, "A global stability result in network flow control," Systems and Control Letters, vol. 46, pp. 165-172, 2002.

[8] E. Altman, T. Başar, and R. Srikant, "Robust rate control for ABR sources," in Proceedings of IEEE INFOCOM 1998, San Francisco, CA, 1998, pp. 166-173.

[9] C. Hollot and Y. Chait, "Nonlinear stability analysis for a class of TCP/AQM networks," in Proceedings of the IEEE Conference on Decison and Control, Orlando, FL, Dec. 2001, pp. 2309-2314.

[10] S. Kunniyur and R. Srikant, "A time-scale decomposition approach to adaptive ECN marking," IEEE Transactions on Automatic Control, vol. 47, no. 6, pp. 884-894, June 2002.

[11] A. J. van der Schaft, $\mathcal{L}_{2}$-gain and Passivity Techniques in Nonlinear Control, 2nd ed. New York and Berlin: SpringerVerlag, 2000.

[12] B. Anderson and S. Vongpanitlerd, Network Analysis and Synthesis - A Modern Systems Theory Approach. New Jersey: PrenticeHall, 1973.

[13] R. Benhabib, R. Iwens, and R. Jackson, "Stability of distributed control for large flexible structures using positivity concepts," in AIAA Guidance and Control Conference, Paper No. 79-1780, Boulder, Co., Aug. 1979.

[14] G. Zames, "On the input/output stability of time-varying nonlinear feedback systems, part I: Conditions derived using concepts of loop gain, conicity, and positivity, part II: Conditions involving circles in the frequency plane and sector nonlinearities," IEEE Transactions on Automatic Control, vol. 11, no. 3, pp. 465-476, 1966.

[15] J. Willems, "Dissipative dynamical systems, part I: General theory, part II: Linear systems with quadratic supply rate," Arch. Rational Mech. Anal., vol. 45, pp. 321-393, 1972.

[16] H. Khalil, Nolinear Systems, 3rd ed. Prentice-Hall, 2002.

[17] F. Paganini, J. Doyle, and S. Low, "Scalable laws for stable network congestion control," in Proceedings of 2001 Conference on Decision and Control, Orlando, FL, Dec. 2001, pp. 185-190.

[18] J. Willems, Stability Theory of Dynamical Systems. New-York: Wiley, 1970.

[19] G. Franklin, J. Powell, and A. Emami-Naeini, Feedback Control of Dynamic Systems, 3rd ed. Addison-Wesley, 1994.

[20] J. Wen and M. Arcak, "A unifying passivity framework for network flow control," ECSE Dept., Rensselaer Polytechnic Institute, Troy, NY, Technical Report, Aug. 2002.

[21] G. Zames and P. L. Falb, "Stability conditions for systems with monotone and slope-restricted nonlinearities," SIAM J. Control, vol. 6, pp. 89-109, 1968.

[22] S. Kunniyur and R. Srikant, "End-to-end congestion control: Utility functions, random losses and ecn marks," in Proceedings of INFOCOM 2000, Tel Aviv, Israel, Mar. 2000.

[23] P. Ioannou and G. Tao, "Frequency domain conditions for strictly positive real functions," IEEE Trans. on Automatic Control, vol. 32, no. 10, pp. 53-54, Oct 1987.

[24] J. Wen, "Time domain and frequency domain conditions for strict positive realness," IEEE Trans. on Automatic Control, vol. 33, no. 10 , pp. $988-992$, Oct 1988. 